\title{
Gap junctions in isolated rat aorta: evidence for contractile responses that exhibit a differential dependence on intercellular communication
}

G.J. Christ ${ }^{1}$ and P.R. Brink ${ }^{2}$

\section{Correspondence \\ G.J. Christ \\ Laboratory of Molecular and \\ Integrative U rology \\ Institute for Smooth Muscle Biology \\ Albert Einstein College of Medicine \\ Room 744, Forchheimer Building \\ 1300 Morris Park Avenue \\ Bronx, NY 10461 \\ USA \\ Fax: + 1-718-430-8983/828-2705 \\ E-mail: christ@ aecom.yu.edu}

Presented at the Meeting

"Gap Junctions in the Nervous and

Cardiovascular Systems: Clinical

Implications", Rio de Janeiro, RJ,

Brazil, June 6-11, 1998.

Research supported in part by USPHS grants DK46379 to G.J. Christ and GM 56263 to P.R. Brink.

Received August 13, 1999 Accepted September 15, 1999

\author{
${ }^{1}$ Departments of Urology and Physiology and Biophysics, \\ Albert Einstein College of Medicine, Bronx, NY, USA \\ 2Department of Physiology and Biophysics, SUNY at Stony Brook, \\ Stony Brook, NY, USA
}

\section{Abstract}

Connexin43 $(\mathrm{Cx} 43)$ is a major gap junction protein present in the Fischer-344 rat aorta. Previous studies have identified conditions under which selective disruption of intercellular communication with heptanol caused a significant, readily reversible and time-dependent diminution in the magnitude of $\alpha_{1}$-adrenergic contractions in isolated rat aorta. These observations have indentified a significant role for gap junctions in modulating vascular smooth muscle tone. The goal of these steady-state studies was to utilize isolated rat aortic rings to further evaluate the contribution of intercellular junctions to contractions elicited by cellular activation in response to several other vascular spasmogens. The effects of heptanol were examined $(0.2-2.0 \mathrm{mM})$ on equivalent submaximal ( $\approx 75 \%$ of the phenylephrine maximum) aortic contractions elicited by 5-hydroxytryptamine (5-HT; 1-2 $\mu \mathrm{M})$, prostaglandin $\mathrm{F}_{2 \alpha}\left(\mathrm{PGF}_{2 \alpha} ; 1 \mu \mathrm{M}\right)$ and endothelin-1 (ET-1; $\left.20 \mathrm{nM}\right)$. Statistical analysis revealed that $200 \mu \mathrm{M}$ and $500 \mu \mathrm{M}$ heptanol diminished the maximal amplitude of the steady-state contractile responses for 5-HT from a control response of $75 \pm 6 \%(\mathrm{~N}=26$ rings $)$ to $57 \pm 7 \%$ $(\mathrm{N}=26$ rings) and $34.9 \pm 6 \%(\mathrm{~N}=13$ rings $)$, respectively $(\mathrm{P}<0.05)$, and for $\mathrm{PGF}_{2 \alpha}$ from a control response of $75 \pm 10 \%(\mathrm{~N}=16$ rings $)$ to $52 \pm$ $8 \%(\mathrm{~N}=19$ rings $)$ and $25.9 \pm 6 \%(\mathrm{~N}=18$ rings $)$, respectively $(\mathrm{P}<0.05)$. In contrast, $200 \mu \mathrm{M}$ and $500 \mu \mathrm{M}$ heptanol had no detectable effect on the magnitude of ET-1-induced contractile responses, which were 76 $\pm 5.0 \%$ for the control response ( $\mathrm{N}=38$ rings), $59 \pm 6.0 \%$ in the presence of $200 \mu \mathrm{M}$ heptanol $(\mathrm{N}=17$ rings $)$, and $70 \pm 6.0 \%$ in the presence of $500 \mu \mathrm{M}$ heptanol $(\mathrm{N}=23$ rings $)(\mathrm{P}<0.13)$. Increasing the heptanol concentration to $1 \mathrm{mM}$ was associated with a significant decrease in the magnitude of the steady-state ET-1-induced contractile response to $32 \pm 5 \%$ (21 rings; $\mathrm{P}<0.01)$; further increasing the heptanol concentration to $2 \mathrm{mM}$ had no additional effect. In rat aorta then, junctional modulation of tissue contractility appears to be agonist-dependent.
Key words

- Gap junctions

- Connexin43

- Rat aorta

- Vascular smooth muscle

- Intercellular communication 


\section{Introduction}

A common feature of vasculature in diverse species is a perivascular autonomic innervation confined to the adventitial-medial smooth muscle border, with vascular smooth muscle cells that lack electrical excitability (i.e., the absence of regenerative electrical events) (1-5). The implication of such observations is that another mechanism is required for the coordination of vasomotor tone. In this regard, aqueous intercellular channels, referred to as gap junctions, are known to interconnect adjacent cells in most tissues including vascular smooth muscle (3-18). Gap junctions provide partial cytoplasmic continuity between coupled cells, and thus serve as an ideal anatomic substrate for coordinating tissue responses $(5,13,19)$. In fact, recent studies on isolated vascular tissues and cultured smooth muscle cells clearly indicate the importance of gap junctions to coordination of tissue responses among vascular smooth muscle cells $(5,8,10-13,16)$, including the vascular smooth muscle cells of large conduit vessels (e.g., aorta) $(6,10,14)$.

From a physiological standpoint, intercellular communication among aortic vascular smooth muscle cells might be expected to affect cardiovascular homeostasis. For example, the tone of the aorta is critical to the impedance matching of cardiac output, ensuring the most efficient transmission of blood volume from the left ventricle to the peripheral blood vessels (see Ref. 1 for review). As such, coordinated aortic smooth muscle responses would presumably be an important determinant of aortic compliance, and thus, of the transient accommodation characteristics of the aorta (1). The gap junction protein connexin 43 is a major gap junction protein present between vascular smooth muscle cells in the sparsely innervated rat aorta $(6,10,14)$. Moreover, selective disruption of intercellular communication with the lipophilic compound heptanol (at concentrations $\leq 500 \mu \mathrm{M}$ ) significantly diminishes both the rate and magnitude of $\alpha_{1}$-adrenergic receptor-mediated contractile responses in aorta. As such, in rat aorta, gap junctions may play a major role in modulating contractile responses following receptor activation $(6,10,14)$.

However, in addition to activation of $\alpha_{1}$-adrenergic receptors, other neurotransmitters, neuromodulators and hormones are known to contract isolated aortic rings via receptor-mediated mechanisms. With this in mind, the goal of the present investigation was to evaluate the potential contribution of gap junctions to contractions elicited by other physiologically relevant spasmogens. This report demonstrates that preincubation of aortic rings with heptanol caused a doserelated and agonist-dependent diminution in the contractile responses elicited by all three agonists tested, namely, endothelin-1 (ET1), 5-hydroxytryptamine (5-HT) and prostaglandin $\mathrm{F}_{2 \alpha}\left(\mathrm{PGF}_{2 \alpha}\right)$. Because heptanol, at the concentrations used, has selective pharmacological actions on gap junctions $(6,10$, 14), this report provides additional support for the importance of gap junctions in the modulation of contractile responses mediated by the activation of diverse receptor systems. The significance of this intercellular communication is presumably related to the modulation of vasomotor tone.

\section{Material and Methods}

\section{Tissue preparation and pretreatment}

Male Fischer 344 rats (300-400 g, Taconic Farms, Germantown, PA, USA) were sacrificed by $\mathrm{CO}_{2}$ asphyxiation and a few equal length $(\approx 5 \mathrm{~mm})$, endothelium-denuded aortic rings were prepared as previously described $(6,10,14)$. Briefly, aortic rings were placed in $20 \mathrm{ml}$ organ baths maintained at 37 $\pm 0.05^{\circ} \mathrm{C}$, containing Kreb's bicarbonate buffer of the following composition: $124 \mathrm{mM}$ $\mathrm{NaCl}, 5 \mathrm{mM} \mathrm{KCl}, 1.3 \mathrm{mM} \mathrm{MgSO}_{4}, 2.5 \mathrm{mM}$ $\mathrm{CaCl}_{2}, 0.6 \mathrm{mM} \mathrm{KH}{ }_{2} \mathrm{PO}_{4}, 25 \mathrm{mM} \mathrm{NaHCO}$, 
and $10 \mathrm{mM}$ glucose, bubbled with $95 \% \mathrm{O}_{2}-$ $-5 \% \mathrm{CO}_{2}$, at a constant $\mathrm{pH}$ of $7.5 \pm 0.1$. Tissue tension was initially set at $3 \mathrm{~g}$, and when resting tension reached a stable baseline (60-90 $\mathrm{min})$, tension was readjusted to 2.0 g. Contractions were measured isometrically with a Grass Force Displacement Transducer (Model FT-03, Quincy, MA, USA) and recorded on a Grass Polygraph (Model 7D). All tissues were prepared as described above, except in the case of 5-HT where the tissue baths were pretreated with the following substances prior to experimentation: pargyline (100 $\mu \mathrm{M}$ for $30 \mathrm{~min}$ ) to prevent enzymatic degradation of 5-HT, and hydrocortisone (10 $\mu \mathrm{M}$ for $30 \mathrm{~min})$ and cocaine $(3 \mu \mathrm{M}$ for $10 \mathrm{~min}$ ) to prevent non-neuronal and neuronal uptake of 5-HT, respectively.

\section{Experimental design and statistical analysis}

For comparative purposes, and to control for inter-tissue variability of contractile responses, all tissues were equivalently contracted to approximately $75 \%$ of their respective maximal phenylephrine (PE)-induced contractile response, with 5-HT (1-2 $\mu \mathrm{M})$, $\mathrm{PGF}_{2 \alpha}(1 \mu \mathrm{M})$ or ET-1 (20 nM) (see Results). In order to do so, the maximal PE-induced contractile responses were determined on every ring (i.e., the contractile response to application of $10 \mu \mathrm{M} \mathrm{PE}$ ). The specific experimental protocols are described below, but in all cases each aortic ring was exposed to only one agonist, and one, if any, heptanol concentration.

All statistical analyses were performed using the Statview II software package (Abacus Concepts, Berkeley, CA, USA). The effects of distinct heptanol concentrations (i.e., 200 or $500 \mu \mathrm{M}$ ) on 5-HT-, PGF $_{2 \alpha^{-}}$and ET-1-induced contractions were initially assessed by two-factor analysis of variance. Post-hoc multiple comparisons were then performed as appropriate when the one-factor analysis of variance was significant at $\mathrm{P}<0.05$. The protected Fisher least signifi- cant difference test (PLSD) was utilized to assess the significance of all pairwise comparisons. A Student $t$-test for unpaired samples was utilized to evaluate the statistical significance of higher heptanol concentrations on ET-1-induced contractile responses.

\section{Experimental protocol for 5-HT, $\mathrm{PGF}_{2 \alpha^{-}}$and ET-1-induced contractions}

For 5-HT and $\mathrm{PGF}_{2 \alpha}$, elicitation of a control contractile response was followed by a single preincubation of randomly selected rings with heptanol (either 200 or 500 $\mu \mathrm{M}$ ) for $10-15 \mathrm{~min}$, prior to a second addition of either 5-HT or $\mathrm{PGF}_{2 \alpha}$. These heptanol concentrations were selected based on previous experience with the heptanol sensitivity of this and other preparations $(6,10,14,16)$. Control rings (aortic rings that were never exposed to heptanol, but merely contracted twice by the same concentration of agonist) were run in parallel to assess the possibility of time-dependent alterations in tissue sensitivity to 5-HT and/or $\mathrm{PGF}_{2 \alpha}$ (e.g., either desensitization or supersensitivity). However, due to the rather long-lasting nature of the ET-1-induced contractile response the experimental protocol was slightly different (i.e., it frequently took 1-2 $\mathrm{h}$ for the ET-1-induced contractile response to return to resting tension levels following a single application and repeated washouts with drug-free Kreb's buffer). Therefore, with respect to ET-1, all rings were subjected to a single exposure to ET-1 with control and experimental rings randomly selected as described above. After preincubation of some rings with heptanol, ET-1 was added to all tissues. That is, all rings were subjected to a single exposure to ET-1, in either the presence or absence of heptanol. Since there were no significant alterations in ET-1-induced contractility in the presence of 200 or $500 \mu \mathrm{M}$ heptanol, a separate series of experiments was conducted to examine the effects 
of tissue preincubation with even higher heptanol concentrations (e.g., 1-2 mM).

\section{Results}

\section{Effects of 200 and $500 \mu \mathrm{M}$ heptanol on 5-HT-, PGF $2 \alpha^{-}$and ET-1-induced contractions in isolated rat aorta}

A two-factor ANOVA revealed that preincubation with heptanol significantly diminished the magnitude of steady-state contractions in a dose-related $(\mathrm{P}<0.007)$ and agonist-dependent $(\mathrm{P}<0.0001)$ fashion, with a significant agonist-heptanol interaction $(\mathrm{P}<0.005)$. A one-factor ANOVA with post hoc multiple comparisons of observed responses (expressed as the mean \pm SEM) among the control, $200 \mu \mathrm{M}$ and $500 \mu \mathrm{M}$ treatment groups was then performed for each agonist. A representative example of our observations with each agonist is illustrated in Figure 1. The results of statistical analyses are summarized in Table 1 and graphically depicted in Figure 2.

Briefly, preincubation with $200 \mu \mathrm{M}$ heptanol produced a significant reduction in the magnitude of both 5-HT- and $\mathrm{PGF}_{2 \alpha}$-induced steady-state contractile responses $(\mathrm{P}<0.05)$. Moreover, increasing the heptanol concentration in the bath to 500 $\mu \mathrm{M}$ was associated with a further decrease in the magnitude of the steady-state contractile response to both agonists $(\mathrm{P}<0.05)$. In stark contrast, preincubation with 200 or $500 \mu \mathrm{M}$ heptanol, respectively, had no significant effect on the magnitude of an equivalent ET-1-induced steady-state contractile response $(\mathrm{P}<0.13)$. In order to further explore the dependence of ET-1-induced contractile responses on intercellular communication through gap junctions, some aortic rings were preincubated with $1 \mathrm{mM}$ heptanol. At this bath concentration, a significant decrease in the magnitude of the steady-state ET-1-induced contractile response to $32 \pm 5 \%$ was observed ( 21 rings; $\mathrm{P}<0.01$, Student $t$-test for unrelated samples; compare with control levels in Table 1). Increasing the heptanol concentration in the bath to $2 \mathrm{mM}$ caused no further reduction in the ET-1-induced response.

\section{Discussion}

The exact mechanism of action of heptanol is still unknown, and certainly this is a most important consideration relative to the current observations. In this regard, an algorithm for identifying reasonable experimental conditions under which heptanol may be utilized as a "relatively selective" uncoupling agent has been reported in recent publications $(6,10,14,16)$, and will not be fur-
Figure 1 - Time course of response generation for endothelin-1 (ET-1)-, prostaglandin $\mathrm{F}_{2 \alpha}$ $\left(\mathrm{PGF}_{2 \alpha}\right)$ - and 5-hydroxytryptamine (5-HT)-induced contractions in the presence of $0.5 \mathrm{mM}$ heptanol (Hept.) (panel A). Panel $B$ depicts the effects of higher heptanol concentrations on ET1-induced contractions. Each tracing illustrates representative contractile responses measured at 1-min intervals in a single (distinct) aortic ring. PE, Phenylephrine.
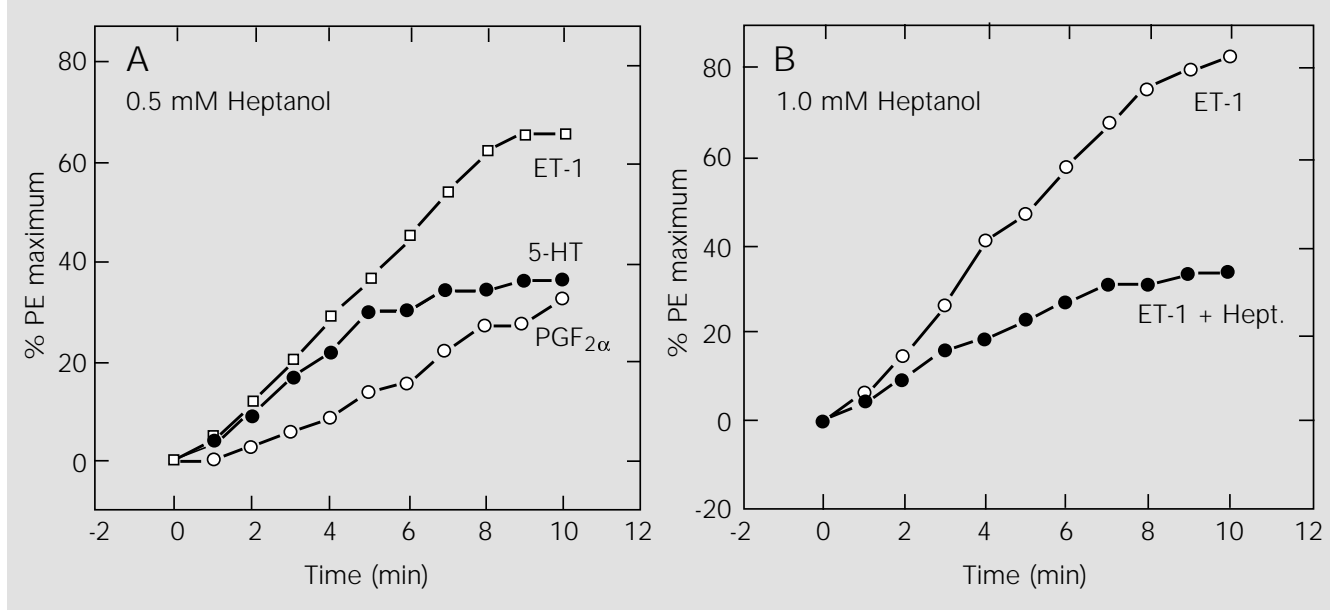
ther reviewed here. More recently, it was clearly shown that the ability of heptanol to elicit relaxation of PE-precontracted, but not $\mathrm{KCl}$-precontracted, rat aortic rings was timedependent (6), and in addition, independent of the order of addition of PE or heptanol (Christ GJ, Spektor M and Brink PR, unpublished observations). Specifically, immediately following achievement of a steadystate PE-induced contractile response, application of heptanol to aortic rings produces a $\approx 50 \%$ loss of measured tension (i.e., relaxation). For all subsequent time points up to 40 min after the addition of PE, the longer the elapsed time interval between addition of PE to the aortic rings and the application of heptanol, the smaller the observed relaxation response. Consistent with this latter result, linear regression analysis revealed a highly significant negative correlation between this time interval and percent relaxation of PE-precontracted aortic rings. In fact, $\approx 40$ min after the addition of PE, the application of heptanol produced little or no detectable effect on the magnitude of the PEinduced steady-state contractile response. In addition, at no point in time did heptanol ever elicit a detectable relaxation response in KCl-precontracted tissues, even when the measured tension was as low as $600 \mathrm{mg}$. Certainly, these observations provide the most compelling demonstration yet that heptanol can indeed have selective uncoupling actions. Therefore, for the purposes of the following discussion we will assume that the major effect of heptanol on tension development in the rat aortic rings is related to a relatively selective action on the intercellular junctions.

As such, the major finding of these new studies is a differential sensitivity of equivalently precontracted aortic rings to activation of distinct membrane receptor-effector systems, namely, those mediated by application of $\mathrm{PGF}_{2 \alpha}, 5-\mathrm{HT}$ and ET-1. In this regard, these observations are quite consistent with, and therefore complement our previous stud- ies which documented that the partial $\alpha_{1}$ adrenergic agonist oxymetazoline was more sensitive to the uncoupling effects of heptanol than the higher efficacy $\alpha_{1}$-adrenergic ago-

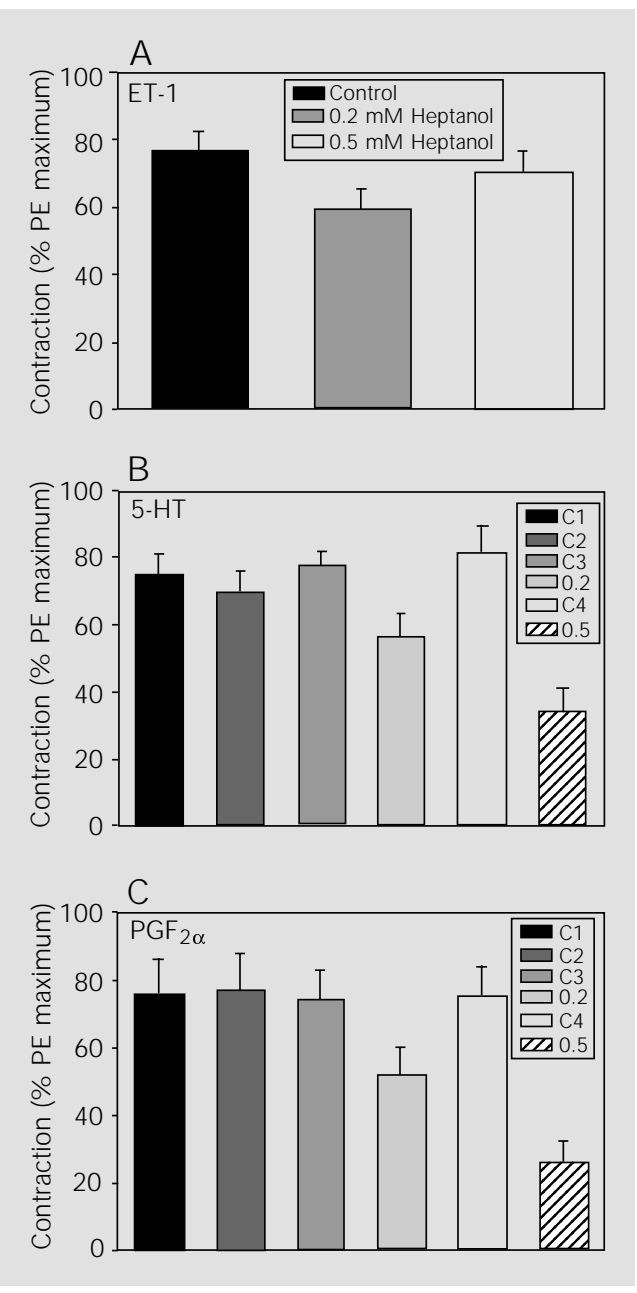

Figure 2 - Graphic depiction of heptanol-induced alterations in agonist-induced contractions. Shown are the mean \pm SEM responses observed under the following conditions: Panel A, Control ET-1-induced contractile response $(20 \mathrm{nM})$, and the response observed following preincubation with 0.2 and $0.5 \mathrm{mM}$ heptanol, respectively (see $\mathrm{Ma}$ terial and Methods for details). In Panel B (5-HT; 1-2 $\mu \mathrm{M})$ and Panel C ( $\left.\mathrm{PGF}_{2 \alpha} ; 1 \mu \mathrm{M}\right), \mathrm{C} 1$ and $\mathrm{C} 2$ denote the paired time-dependent control response to two successive applications of agonist to the same aortic ring (see Material and Methods); C3 and 0.2 denote the paired responses of the same aortic ring to the application of the same concentration of agonist on the same ring in the absence (C3) and presence (0.2) of $0.2 \mathrm{mM}$ heptanol; $\mathrm{C} 4$ and 0.5 denote the paired responses of the same aortic ring to the application of the same concentration of agonist on the same ring in the absence (C4) and presence (0.5) of $0.5 \mathrm{mM}$ heptanol. The number of observations, statistical significance levels and actual values for all agonists are displayed in Table 1. For abbreviations, see legend to Figure 1.

Table 1 - Effects of heptanol on the steady-state magnitude of the $\mathrm{PGF}_{2 \alpha^{-}}, 5-\mathrm{HT}$ - and ET-1-induced contractile responses in equivalently contracted isolated rat aortic rings.

The time-dependent control values, that is, the response of rat aortic rings to two successive applications of agonist were: $76 \pm 10 \%(\mathrm{~N}=16)$ and $77 \pm 11 \%$ for $\mathrm{PGF}_{2 \alpha}$, and $75 \pm 6 \%(\mathrm{~N}=25)$ and $70 \pm 6 \%$ for $5-\mathrm{HT}$. Note that two successive applications were not possible for ET-1; see Material and Methods. ${ }^{*} \mathrm{P}<0.05$ compared to control (Fischer's PLSD post hoc analysis). For abbreviations, see legend to Figure 1.

\begin{tabular}{lcccc}
\hline Drug & $\begin{array}{c}\text { Control } \\
(\%)\end{array}$ & $\begin{array}{c}0.2 \mathrm{mM} \text { Heptanol } \\
(\%)\end{array}$ & $\begin{array}{c}\text { Control } \\
(\%)\end{array}$ & $\begin{array}{c}0.5 \mathrm{mM} \text { Heptanol } \\
(\%)\end{array}$ \\
\hline $\mathrm{PGF}_{2 \alpha}$ & $74 \pm 9(\mathrm{~N}=19)$ & $52 \pm 8^{*}$ & $75 \pm 9(\mathrm{~N}=19)$ & $26 \pm 6^{*}$ \\
$5-\mathrm{HT}$ & $77 \pm 5(\mathrm{~N}=25)$ & $57 \pm 7^{*}$ & $82 \pm 8(\mathrm{~N}=13)$ & $35 \pm 7^{*}$ \\
$\mathrm{ET}-1$ & $77 \pm 5(\mathrm{~N}=38)$ & $59 \pm 6(\mathrm{~N}=17)$ & $77 \pm 5(\mathrm{~N}=38)$ & $70 \pm 6(\mathrm{~N}=23)$
\end{tabular}


nist phenylephrine at the same two heptanol concentrations, i.e., 200 and $500 \mu \mathrm{M}$ (10). What then might be the explanation for such agonist- and efficacy-dependent differences in heptanol sensitivity? Some possibilities are reviewed below.

In these studies, it is assumed that heptanol is homogeneously distributed in the cell membrane, and furthermore that at the concentrations used the predominant action of heptanol is to cause selective disruption at the junctional-lipid interface by intercalating in the lipid bilayer and thus effectively gating the channel closed $(6,10,14,16)$. In this scenario, the proportion of affected channels is assumed to be some constant fraction of the heptanol concentration $(6,10,14)$. As such, the agonist-dependent disparities in heptanol sensitivity are hypothesized to reflect fundamental characteristics of second messenger formation/junctional permeability that might, at least in part, contribute to the documented differences in agonist efficacy.

One possibility, for example, is that the observed differential heptanol sensitivity may be due to an agonist-dependent accessibility of intercellular channels and/or a greater second messenger driving force or cellular sensitivity to activation. As such, an agonist such as ET-1 would be hypothesized to essentially have a greater "gap junction reserve" 5-HT or $\mathrm{PGF}_{2 \alpha}$. It follows then that for every quantum of receptor activation with an agonist like ET-1, more cells that are not directly activated by agonist would "see" the effects of cellular activation indirectly by diffusion of messenger molecules through gap junctions. Such a hypothesis is consistent with studies performed on rat aortic rings in which it was clearly demonstrated that for any given level of intracellular calcium increase there was a greater level of myosin phosphorylation than that observed with, for example, PGF $_{2 \alpha}$ (20). Furthermore, for any given level of myosin phosphorylation, ET1 -induced contractile responses are associated with the greater levels of tension development. In this context, such observations are consistent with the supposition that intercellular calcium/ $/ \mathrm{IP}_{3}$ diffusion may be a primary mechanism for propagating/coordinating vascular smooth muscle responses (15). Therefore, there are several independent lines of investigation that support the hypothesis that ET-1-induced contractions, by virtue of the apparently greater cellular sensitivity their activation invokes, may be more gap junction independent than contractions of equivalent magnitude produced by other agonists.

In conclusion, this study provides additional support for a role of gap junctions in the modulation of aortic contractile responses mediated by the activation of diverse receptor systems. Moreover, in rat aorta, junctional modulation of tissue contractility appears to be both agonist- and efficacy-dependent. The physiological significance of such differential agonist dependence on intercellular communication is presumably related to the dynamic physiologic/pathophysiologic modulation of vascular tone. However, there is no doubt that further molecular, biochemical and electrophysiological studies are necessary in order to gain better insight into the mechanism(s) governing such agonist- and efficacy-dependent differential modulation of intercellular communication in this and other vascular tissues.

\section{Acknowledgments}

We gratefully acknowledge the technical assistance of Marjorie Gondré, Dr. Brian Davis-Joseph and Weixin Zhao. 


\section{References}

1. Gibbins IL, Morris J L, Fumess J B \& Costa $M$ (1987). Nonadrenergic Innervation of Blood Vessels. CRC Press, Boca Raton, $\mathrm{FL}, 1-36$

2. Bevan J A \& Torok J (1970). Movement of norepinephrine through the media of rabbit aorta. Circulation Research, 27: 325331.

3. Bumstock $G$ (1970). Structure of smooth muscle and its innervation. In: Bulbring $E$, Brading AF, J ones AW \& Tomita T (Editors), Smooth Muscle. Williams \& Wilkins, Baltimore, MD, 1-69.

4. Hirst GDS \& Edwards FR (1989). Sympathetic neuroeffector transmission in arteries and arterioles. Physiological Reviews, 69: 546-604.

5. Christ GJ , Spray DC, El-Sabban M, Moore LK \& Brink PR (1996). Gap junctions in vascular tissues. Evaluating the role of intercellular communication in the modulation of vasomotor tone. Circulation Research, 79: 631-646.

6. Christ GJ , Spektor M, Brink PR \& Barr L (1999). Further evidence for the selective disruption of intercellular communication by heptanol. American J ournal of Physiology, 276: H1911-H1917.

7. Christ GJ \& Brink PR (1999). An analysis of the presence and physiological relevance of subconducting states of connexin43-derived gap junction channels in cultured human corporal vascular smooth muscle cells. Circulation Research, 84: 797-803.

8. Brink PR, Ramanan SV \& Christ GJ (1996). Human connexin 43 gap junction channel gating: evidence for mode shifts and/or heterogeneity. American J oumal of Physiology, 271: C321-C331.

9. Chaytor AT, Evans WH \& Griffith TM (1997). Peptides homologous to extracellular loop motifs of connexin 43 reversibly abolish rhythmic contractile activity in rabbit arteries. Journal of Physiology, 503: 99-110.

10. Christ GJ (1995). Modulation of $\alpha_{1}$-adrenergic contractility in isolated vascular tissues by heptanol: a functional demonstration of the potential importance of intercellular communication to vascular response generation. Life Sciences, 56: 709-721.

11. Christ GJ (1997). The "syncytial tissue triad": a model for understanding how gap junctions participate in the local control of penile erection. World J oumal of Urology, 15: 36-44.

12. Christ GJ , Brink PR, Melman A \& Spray DC (1993). The role of gap junctions and ion channels in the modulation of electrical and chemical signals in human corpus cavemosum smooth muscle. International J oumal of Impotence Research, 5: 77-96.

13. Christ GJ , Brink PR \& Ramanan SV (1994). Dynamic gap junctional communication: a delimiting model for tissue responses. Biophysical J oumal, 67: 1335-1344.

14. Christ GJ, Brink PR, Zhao W, Moss J, Gondre CM, Roy C \& Spray DC (1993). Gap junctions modulate tissue contractility and $\alpha_{1}$-adrenergic agonist efficacy in isolated rat aorta. J ournal of Pharmacology and Experimental Therapeutics, 266: 1054-1065.

15. Christ GJ , Moreno AP, Melman A \& Spray
DC (1992). Gap junction-mediated intercellular diffusion of $\mathrm{Ca}^{2+}$ in cultured human corporal smooth muscle cells. American J ournal of Physiology, 263: C373C383.

16. Christ GJ , Moreno AP, Parker ME, Gondre $C M$, Valcic M, Melman A \& Spray DC (1991). Intercellular communication through gap junctions: a potential role in pharmacomechanical coupling and syncytial tissue contraction in vascular smooth muscle isolated from the human corpus cavernosum. Life Sciences, 49: L195L200.

17. Watts SW, Tsai ML, Loch-Caruso $R \&$ Webb RC (1994). Gap junctional communication and vascular smooth muscle reactivity: use of tetraethylammonium chloride. J ournal of Vascular Research, 31: 307-313.

18. Watts SW \& Webb RC (1996). Vascular gap junctional communication is increased in mineralocorticoid-salt hypertension (see comments). Hypertension, 28: 888-893.

19. Ramanan SV, Brink PR \& Christ GJ (1998). Neuronal innervation, intracellular signal transduction and intercellular coupling: A model for syncytial tissue responses in the steady state. J oumal of Theoretical Biology, 193: 69-84.

20. Karaki H (1991). $\mathrm{Ca}^{2+}$ regulation of vascular smooth muscle and release of endothelium-derived relaxing factor. In: Sperelakis N \& Kuriyama H (Editors), Ion Channels of Vascular Smooth Muscle Cells and Endothelial Cells. Elsevier, New York, 297-315. 